\title{
Fluorescence lifetime assisted enhanced security feature in travel documents for border control and security applications.
}

JOHNY, J., OFFICER, S., FUNG, W.K., PRABHU, R. be made for personal use only. Systematic reproduction and distribution, duplication of any material in this publication for a fee or for commercial purposes, and modification of the contents of the publication are prohibited. 


\title{
Fluorescence lifetime assisted enhanced security feature in travel documents for border control and security applications
}

\author{
Jincy Johny ${ }^{1}$, Simon Officer ${ }^{2}$, Wai Keung Fung1, Radhakrishna Prabhu ${ }^{1}$ \\ ${ }^{1}$ School of Engineering, Robert Gordon University, Aberdeen, United Kingdom \\ ${ }^{2}$ School of Pharmacy and Life Sciences, Robert Gordon University, Aberdeen, United Kingdom
}

\begin{abstract}
Border management and security challenges are increasing considerably in recent years. One of the major concerns is counterfeiting and fraudulent use of identity and other travel documents for crossing border controls. This poses serious threats and safety concerns worldwide, considering the scenario of terrorism and illegal migration across the world. Hence, advanced technologies with improved security features becomes essential to strengthen border security and to enable smooth transits. In this paper, we present a novel dual waveguide based invisible fluorescence security feature with lifetime discrimination and a simple validation system. Molecular fluorescence and lifetimes from the rare earth doped waveguides can be used as additional security features in the identity documents. The validation system consists of a modulated excitation source and fast photo-diodes which helps in the simultaneous detection of multiple security features from the fluorescence waveguides. The rare earth doped fluorescence waveguides are embedded into the identity document as micro-threads or tags which are invisible to the naked eye and are only machine readable. Rare earth fluorescence materials have higher sensitivity and selectivity as they absorb only specific ultraviolet (UV) or visible (VIS) wavelengths to create corresponding fluorescent emissions in the visible or infrared wavelengths. Herein, we present the results based on the fluorescence and fluorescence lifetime spectroscopic studies carried out on the terbium (Tb) and dysprosium (Dy) doped waveguides. The different emission wavelengths and lifetimes of these rare earth elements is a key differentiating feature, providing selectivity and security to the detector systems.
\end{abstract}

Keywords: Fluorescence, Lifetime, Rare earth, Waveguide, Spectroscopy, Security feature, Border control, Anticounterfeiting

\section{INTRODUCTION}

In recent years, border security challenges and immigration issues are increasing considerably, owing to the higher traveler volume, terrorism threats and number of international crimes reported [1]. Counterfeiting and fraudulent use of different identity and travel documents, such as passports [2-3], are posing serious threats and safety concerns worldwide. Although numerous technological developments have emerged for enhancing the security features, such as e-passports [45] and biometrics [6-7], the fraudsters still use different counterfeiting techniques such as cloning, alteration, tampering and misusing of genuine documents, for creating fake documents [8-10]. The advancement in computers, photocopiers, printers and scanners have made counterfeiting and creation of fake documents much easier to the fraudsters. Hence, there arises a need for advanced technologies and improved security features to fight against these fraudulent attacks and to ensure efficient border controls.

Photoluminescent or fluorescent based security methods are promising for adding security features to the valuable printed documents from counterfeiting and other fraud recreations. Moreover, the fluorescence security feature adds authenticity to the printed document [11-12]. Fluorescent dyes are commonly used for labelling and creating visible and invisible indicia or markings on important printed documents. These markings/labeling becomes visible only upon the irradiation of suitable wavelengths of light such as ultraviolet (UV) [13]. Although fluorescent dye-based security marking or labelling is difficult to counterfeit, once the specific dye and its associated spectra is identified it becomes fairly easier for the fraudsters to reproduce or replicate them. This is because dyes have very broad spectra and it is easier to replicate them with alternative compounds. Hence, are earth based photoluminescent nanomaterials such as europium (Eu), thulium $(\mathrm{Tm})$, erbium (Er), ytterbium ( $\mathrm{Yb}$ ), dysprosium (Dy) and terbium (Tb) doped within suitable hosts are emerging as new ink materials for the secure labelling of banknotes and important documents such as passports and visa documents [14] 
During the paper manufacturing process, the rare earth doped waveguide can be easily incorporated by adding the rare earth micro-fibres into the cellulose fibre suspension used for paper making. Important advantages of rare earth materials over other photoluminescent materials such as inks and dyes include: unique excitation-emission patterns, narrow photoluminescence emission profiles, large Stokes shift providing wavelength differentiation and wave guidance properties. The specific fluorescent emission peaks of the rare earth based optical security feature are dependent on the incident light or the excitation wavelength. The rare earth waveguide security feature also provides spectral or color differentiation to swiftly identify the counterfeited document, while authenticating the original ones. Furthermore, the narrow fluorescent emission peaks of rare earth doped materials, enables scope for incorporating multiple rare earth security features within the same printed document. Hence, rare earth fluorescent materials are promising candidates for securing valuable documents against counterfeiting issues, because they are difficult to replicate but easier to authenticate using simple fluorescence spectroscopic techniques. This is because the specific rare earth doped glass composition is difficult to be copied and they also provide unique optical signatures in response to exciting radiation [15].

In this paper, we present a novel dual waveguide-based fluorescence security feature with lifetime discrimination and a simple validation system to enhance the security at border controls. The validation system consists of a modulated excitation/input source and avalanche photodiodes (APDs) integrated with filters which helps in the simultaneous detection of multiple security features from the fluorescence waveguides. The rare earth doped fluorescence waveguides embedded into the identity document as micro-threads or tags are invisible to the naked eye and only machine readable. Rare earth materials absorb only specific ultraviolet or visible wavelengths and create corresponding unique fluorescent emissions in the visible or infrared wavelengths. The spectral security feature from the waveguides along with the advanced fluorescence lifetime security feature makes it impossible for the counterfeiters to try any forging attempts with other dye materials which will have a very different fluorescence and lifetime characteristics. Herein, we present the results based on the fluorescence and fluorescence lifetime spectroscopic studies carried out on the fabricated rare earth doped waveguides. Implementation of these advanced security features will help in stopping fraudsters from crossing international borders, whereas facilitating fast and safe transit for genuine travelers. Furthermore, this waveguide based fluorescent technology can easily work along with other existing security features, serving as an additional security feature within the document. To sum up, the new technique will speed up border control procedures and will overcome the current hassle of multiple manual identification checks.

\section{METHODOLOGY}

\subsection{Working Principle}

The rare earth doped waveguide security feature relies on the unique fluorescence signatures of the rare earth materials doped within the waveguide. Fluorescence or photoluminescence is the light emission caused when a material is irradiated with light (ultraviolet or visible light). Among different light matter interaction phenomenon's including absorption and reflection, photoluminescence is a very important class; as it is a source of new wavelengths of light [16]. Normally, photoluminescence or fluorescence emissions occur at a longer wavelength after a small interval of time (termed as fluorescence lifetime), as a result of the absorption of shorter wavelength (also called the excitation wavelength) [17]. Typically, fluorescence emission occurs within a nanosecond to millisecond time span after light irradiation.


Figure 1. (a) Illustration of energy state transitions (b) excitation and photoluminescence emission profile 
Photoluminescence involves the excitation of electrons from a lower vibrational energy state to a higher energy state with the irradiation of light, from where radiative decay is possible. The optimal or most efficient excitation wavelength usually corresponds to the absorption maximum [18]. During the excitation process, photon absorption happens instantaneously and very soon the photoluminescence molecule relaxes to the lowest vibrational energy level within the excited state. Following this, next transition occurs, and the molecule goes back to its ground state either through spontaneous emission or non-radiative decay process (Figure 1. (a)). In spontaneous emission, energy is released in the form of a photon; whereas in non-radiative decay process, energy is released in the form of heat. The emitted photon has lower energy than excitation photon, as some energy was lost during the relaxation process. This results in a shift in the photoluminescence wavelength with respect to the excitation wavelength and is known as the Stokes shift [19]. Figure 1. (b) shows a typical excitation and emission profile. Usually photoluminescence experiences a red shift relative to the excitation profile, wherein spectral shifting occurs towards the higher wavelength (i.e. lower energy and lower frequency) [20]. The emitted fluorescence from the rare earth doped waveguides can be detected using an array photodetector and used as the security feature. Important advantages of the rare earth waveguide security feature include: unique excitationemission patterns, narrow fluorescence emissions, wavelength differentiation, wave guidance and are invisible and only machine readable.

Fluorescence lifetime is the average time the molecule spends in the excited state before it returns to its ground state usually through fluorescence emission [21]. A complete time resolved spectrum is obtained from the fast photo-diode in a single measurement and the measurement is independent of signal intensity from external forces like absorption, scattering or even concentration [22]. The time domain measurement assumes that when photons are absorbed, the excited molecules move into a brief transition state and subsequent signal emitted are obtained as a measurement of pulse widths [23-24].

\subsection{Waveguide Characterisation}

The fluorescent waveguide is based on $\mathrm{Tb}$ and Dy doped borosilicate glass fabricated though the fibre drawing process [25]. The fluorescence characteristics of the micron sized rare earth doped waveguide was validated using the experimental setup shown in figure 2. The experimental setup used to characterise the dual waveguide configuration embedded into the identity document consisted of UV LED and UV-VIS spectrometer is shown in figure 2. The LED purchased from Nichia Corporation with peak wavelength, $\lambda_{P}=385 \mathrm{~nm}$ and full spectral width at half maximum, FWHM $=15 \mathrm{~nm}$ was used as the light source. The UV irradiation was coupled into the Tb-Dy dual waveguide through a lens assembly and the fluorescence signals were detected from its other end using the StellarNet (EPP2000C) spectrometer.

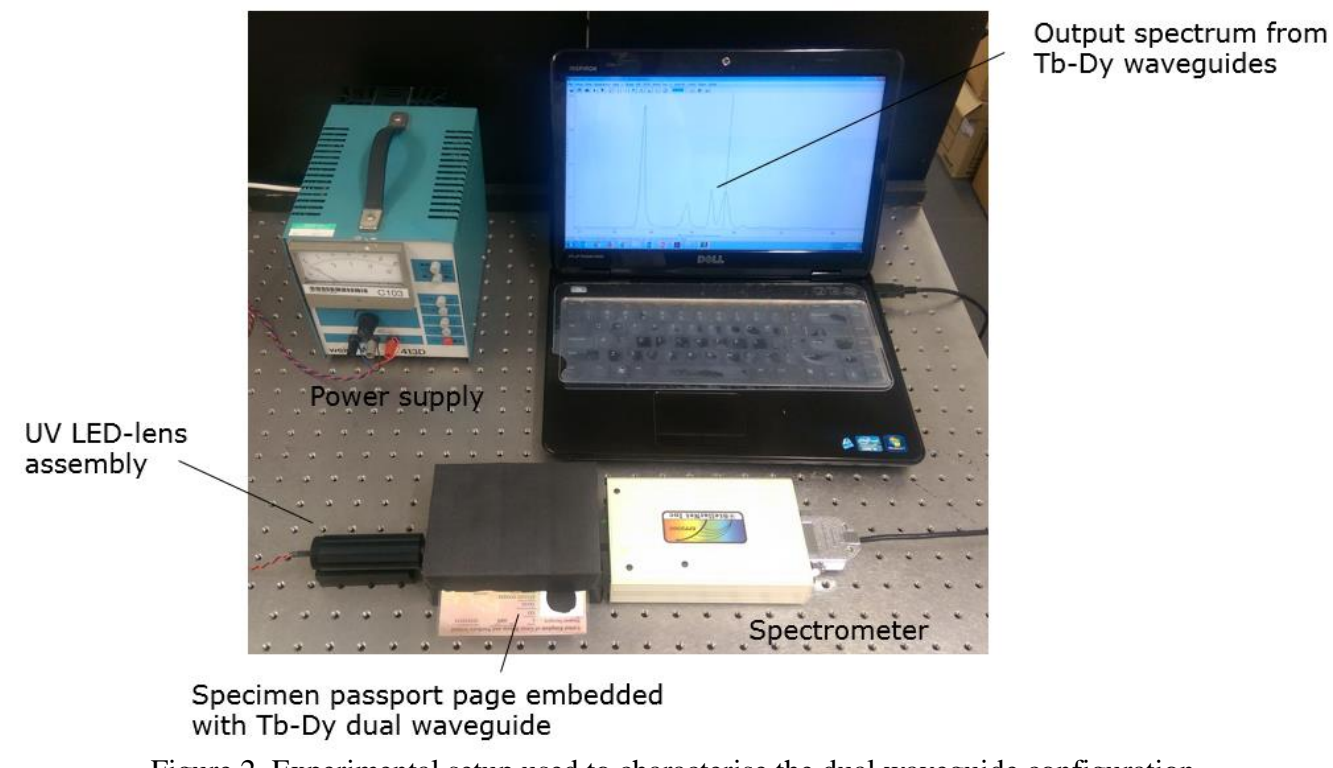

Figure 2. Experimental setup used to characterise the dual waveguide configuration 
Figure 3 shows the image of the prepared sample passport personal information page embedded with Tb-Dy doped dual waveguide security feature.



Figure 3. Sample passport personal information page embedded with Tb-Dy dual waveguide security features

\section{RESULTS AND DISCUSSIONS}

The spectral characterisation of Tb-Dy dual waveguide configuration, both $1.5 \mathrm{~mol} \%$ concentration embedded into the sample passport page was validated using the experimental setup explained in section 2.2. Figure 4 shows the corresponding output spectrum obtained from the dual waveguide when irradiated using $385 \mathrm{~nm}$ UV LED. $385 \mathrm{~nm}$ LED was chosen because, it was identified that both $\mathrm{Tb}$ and Dy samples had highest excitation peaks in this range [25]. As we can observe from the graph, the peak in the $385 \mathrm{~nm}$ region corresponds to the LED excitation peak and the peak around $544 \mathrm{~nm}$ and $575 \mathrm{~nm}$ corresponds to the $\mathrm{Tb}$ and Dy signature peaks. The peak around $485 \mathrm{~nm}$ is present in both $\mathrm{Tb}$ and $\mathrm{Dy}$ samples.



Figure 4. Output spectrum of the $1.5 \mathrm{~mol} \% \mathrm{~Tb}$-Dy dual waveguide

Fluorescence lifetime characterisation of the Tb and Dy doped waveguides were carried out using the FLS 900 Edinburgh Instruments fluorescence lifetime spectrometer. Manual lifetime scanning of the rare earth doped borosilicate glass samples were carried out and following this an exponential fit of the obtained curve gives the fluorescence decay 
time. The excitation and fluorescence emission wavelengths obtained from the UV-VIS absorption and fluorescence spectroscopic studies carried out [25] was used in the lifetime scanning studies.

The most intense excitation peak of $\mathrm{Tb}$ at $376 \mathrm{~nm}$ corresponding to the transition from the Tb ground state ${ }^{7} \mathrm{~F}_{6}$ to the higher excited level $\left({ }^{5} \mathrm{D}_{3},{ }^{5} \mathrm{G}_{6}\right)$ [26], was used as the excitation wavelength in the manual lifetime scanning setting. The Tb emission wavelength was set at $544 \mathrm{~nm}$ corresponding to its characteristic fluorescence green emission $\left({ }^{5} \mathrm{D}_{4} \rightarrow{ }^{7} \mathrm{~F}_{5}\right)$ [27]. The fluorescence lifetime $(\tau)$ of $\mathrm{Tb}$ doped waveguide was obtained as $2.26 \mathrm{msec}$, shown in figure 5 .

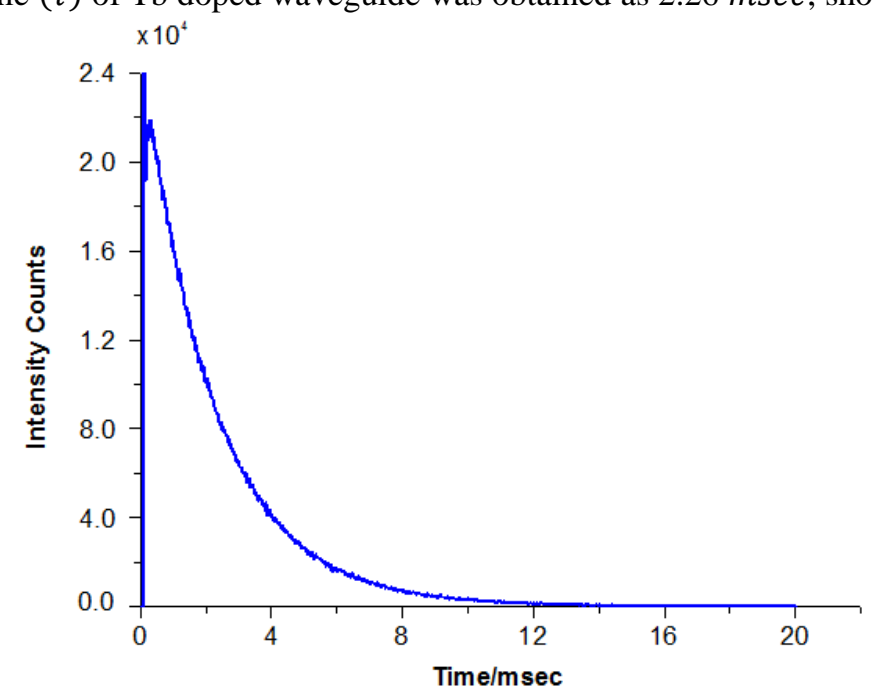

Figure 5. Lifetime graph of $1.5 \mathrm{~mol} \% \mathrm{~Tb}$ doped waveguide

Similarly, the following excitation and emission wavelengths were chosen for the manual lifetime scanning settings of the Dy doped waveguide. The highest intensity $388 \mathrm{~nm}$ excitation peak of Dy corresponding to the transition from the Dy ground state $\left({ }^{6} \mathrm{H}_{15 / 2}\right)$ to the higher energy level $\left({ }^{4} \mathrm{I}_{13 / 2},{ }^{4} \mathrm{~F}_{7 / 2}\right)$. The Dy signature peak was obtained around 575 nm corresponding to its yellowish photoluminescence emission $\left({ }^{4} F_{9 / 2} \rightarrow{ }^{6} H_{13 / 2}\right.$ transition) [28]. The fluorescence lifetime $(\tau)$ of Dy doped waveguide was obtained as $744.63 \mu \mathrm{sec}$ (Figure 6).

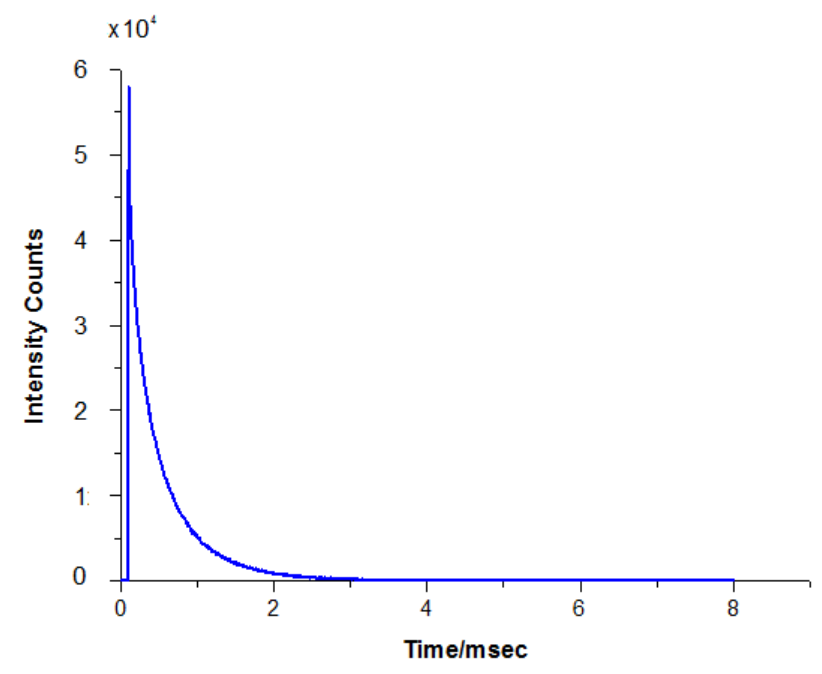

Figure 6. Lifetime graph of $1.5 \mathrm{~mol} \%$ Dy doped waveguide

The differences in the fluorescence lifetimes of $\mathrm{Tb}$ and Dy waveguides is a clear distinguishing feature of rare earth doped materials, which in turn provides higher selectivity and security to the detector systems. Furthermore, the TbDy dual waveguides gives both spectral differentiation and lifetime differentiation. These dual security feature enhances the authenticity of the identity or travel documents, thereby improving the security at the border controls. 


\section{CONCLUSION}

A novel rare earth doped dual waveguide fluorescence security feature with lifetime discrimination and a simple validation system consisting of a modulated excitation source and avalanche photo-diodes is proposed to enhance the security at border controls. The rare earth waveguide security feature can be easily embedded within the travel documents as micron size threads or tags. These invisible fluorescent security features, when irradiated with light, generates specific fluorescent emissions which are unique for each rare earth element. These specific rare earth fluorescence signals are not visible to the naked eye and are only machine readable. Multiple rare earth elements doped into the waveguide provides advanced security features along with high level of authenticity to these identity documents. In addition to improvement of border security control, it facilitates smoother and faster traveler clearances and transits at the ports. Another important advantage of this security feature is that it cannot be easily duplicated using any other reproduction technologies and provides both wavelength and fluorescence lifetime differentiation. Furthermore, this new waveguide fluorescent technique can work in tandem with other existing security features within the document, serving as an additional security feature. The proposed security feature for the travel document is a very simple, cost effective and robust technique. Fluorescence lifetime and spectroscopic characterisation of the fabricated rare earth doped waveguide was carried out to study the lifetime and spectral characteristics of different rare earth elements such as $\mathrm{Tb}$ and $\mathrm{Dy}$. Implementation of these new and advanced machine-readable security features helps in stopping fraudsters from crossing international borders, whereas facilitating fast and safe crossing for genuine travelers.

\section{REFERENCES}

[1] Ackleson J. Directions in border security research. The Social Science Journal. 2003; 40(4):573-581.

[2] Lehtonen P, Aalto P. Smart and secure borders through automated border control systems in the EU? The views of political stakeholders in the Member States. European Security. 2017; 26(2):207-225.

[3] Salter MB. Passports, Mobility, and Security: How smart can the border be? International Studies Perspectives. 2004; 5(1):71-91.

[4] Juels A, Molnar D and Wagner D. Security and Privacy Issues in E-passports. Security and Privacy Issues in Epassports. Security and Privacy for Emerging Areas in Communications Networks, 2005. SecureComm 2005. First International Conference on IEEE; 2005. 74-88.

[5] Schouten B, Jacobs B. Biometrics and their use in e-passports. Image and Vision Computing. 2009; 27(3):305312.

[6] del Rio JS, Moctezuma D, Conde C, de Diego IM, Cabello E. Automated border control e-gates and facial recognition systems. Computers \& Security. 2016; 62:49-72.

[7] Prabhakar S, Pankanti S, Jain AK. Biometric recognition: Security and privacy concerns. IEEE security \& privacy. 2003; (2):33-42.

[8] Lowe P. Counterfeiting: links to organised crime and terrorist funding. Journal of Financial Crime. 2006; 13(2):255-257.

[9] Rudner M. Misuse of passports: identity fraud, the propensity to travel, and international terrorism. Studies in Conflict \& Terrorism. 2008; 31(2):95-110.

[10] Calderoni L, Maio D. Cloning and tampering threats in e-Passports. Expert Systems with Applications. 2014; 41(11):5066-5070.

[11] Kaule W, Schwenk G, Stenzel G. Printed document having a value, with luminescent authenticity feature. 2003.

[12] Ambadiyil S, Krishnendu P, Pillai VM and Prabhu R. Banknote authentication using chaotic elements technology. Banknote authentication using chaotic elements technology. Counterterrorism, Crime Fighting, Forensics, and Surveillance Technologies. International Society for Optics and Photonics; 2017. 1044104.

[13] Dukler S, Landman A. Security documents with visible and invisible markings. 2003.

[14] Officer S, Prabhu GR, Pollard P, Hunter C and Ross GA. Novel online security system based on rare-earthdoped glass microbeads. Novel online security system based on rare-earth-doped glass microbeads. Optical Security and Counterfeit Deterrence Techniques V International Society for Optics and Photonics; 2004. 387 396.

[15] Ross GA, Pollard P, Hunter C, Officer S, Prabhu GR. Optically detectable security feature. 2006; .

[16] Vasconcelos HC, Pinto AS. Fluorescence Properties of Rare-Earth-Doped Sol-Gel Glasses. Recent Applications in Sol-Gel Synthesis. InTech; 2017. 
[17] Albrecht C. Joseph R. Lakowicz: Principles of fluorescence spectroscopy. Analytical and Bioanalytical chemistry. 2008; 390(5):1223-1224.

[18] Albani JR. Structure and dynamics of macromolecules: absorption and fluorescence studies. Elsevier; 2011.

[19] Ishikawa-Ankerhold HC, Ankerhold R, Drummen GP. Advanced fluorescence microscopy techniques-Frap, Flip, Flap, Fret and flim. Molecules. 2012; 17(4):4047-4132.

[20] Sauer M, Hofkens J, Enderlein J. Basic principles of fluorescence spectroscopy. Handbook of fluorescence spectroscopy and imaging: from single molecules to ensembles. $2011 ;: 1-30$.

[21] Alford R, Ogawa M, Hassan M, Gandjbakhche AH, Choyke PL, Kobayashi H. Fluorescence lifetime imaging of activatable target specific molecular probes. Contrast media \& molecular imaging. 2010; 5(1):1-8.

[22] Turconi S, Bingham RP, Haupts U, Pope AJ. Developments in fluorescence lifetime-based analysis for ultraHTS. Drug discovery today. 2001; 6:27-39.

[23] Nkwoada A, Officer S. Fluorescence Lifetime Investigation and Characterization of Dysprosium and Terbium Doped Taggants. Journal of Advanced Chemical Sciences. 2016; :293-295.

[24] Palo K, Brand L, Eggeling C, Jäger S, Kask P, Gall K. Fluorescence intensity and lifetime distribution analysis: toward higher accuracy in fluorescence fluctuation spectroscopy. Biophysical journal. 2002; 83(2):605-618.

[25] Johny J, Bhavsar K, Officer S, Adams M and Prabhu R. Waveguide-based machine readable fluorescence security feature for border control and security applications. Counterterrorism, Crime Fighting, Forensics, and Surveillance Technologies II International Society for Optics and Photonics; 2018. 1080209.

[26] Sontakke AD, Biswas K, Annapurna K. Concentration-dependent luminescence of Tb3 ions in high calcium aluminosilicate glasses. Journal of Luminescence. 2009; 129(11):1347-1355.

[27] Hussain NS, Reddy YP, Buddhudu S. Emission properties of Tb3 -doped zinc boro-silicate glasses. Materials Letters. 2001; 48(5):303-308.

[28] Kaewkhao J, Wantana N, Kaewjaeng S, Kothan S, Kim H. Luminescence characteristics of Dy3 doped Gd2O3$\mathrm{CaO}-\mathrm{SiO} 2-\mathrm{B} 2 \mathrm{O} 3$ scintillating glasses. Journal of rare earths. 2016; 34(6):583-589. 